\title{
Improving Energy Efficiency using a new Game Theory Algorithm for Wireless Sensor Networks
}

\author{
Mahnaz Esmaeeli \\ Department of computer, Dezful Branch, Islamic \\ Azad University, Dezful Iran
}

\author{
Seyed Ali Hosseini Ghahroudi \\ Professor Department of computer, Arsangan \\ Branch, Islamic Azad University, Arsangan Iran
}

\begin{abstract}
In the area of Wireless Sensor Networks (WSNs), improving energy efficiency and network lifetime is one of the most important and challenging issues. On the one hand, the nodes need to stay alive as long as possible by achieving energy efficiency. On the other hand, they have to provide the required service. Energy efficiency based on clustering in wireless sensor networks is one of the most crucial technologies. Game theory has been used recently in a remarkable amount of research in this area. In this paper, we propose an algorithm based on game theory for clustering in wireless sensor networks. In this work our objective is to provide a game theoretical modeling of clustering for wireless sensor networks. Comparing this mechanism to a popular clustering technique, we show via simulations that achieves a performance similar to that of a very popular clustering algorithm.
\end{abstract}

\section{Keywords}

Wireless Sensor Networks, Theory of Games, Clustering, Energy Consumption.

\section{INTRODUCTION}

Wireless Sensor Networks (WSNs) have met a growing interest in the last decade due to their applicability to a large class of contexts, such as environment monitoring, object tracking, traffic control, and health applications, among others. Since it is usually difficult and costly to replace faulty sensors once they are deployed, reducing the energy consumption in WSNs is of paramount importance in order to maximize the network lifetime [1]. The lifetime of a sensor network is defined as the time until any or a given number of sensors in the network dies.

Wireless sensor networks usually have a basic station (sink), which is related with other sensors wirelessly. Each sensor sends its own data to the basic station directly or by the help of other nodes. Then, the collected information of the concerned parameter is processed in the basic station and its exact value is estimated with a relative precision. The clustering-based protocol is one of protocols for prolonging life nodes and reducing energy consumption. In WSN, the sensor nodes are divided into several groups, called cluster. Every cluster would have a leader, often referred to as the cluster-head $(\mathrm{CH})$. In a few WSN scenarios, some high-energy nodes called are deployed in the network. These gateways group sensors to form distinct clusters in the system and act as a $\mathrm{CH}$. The $\mathrm{CH}$ manage the network in the cluster, perform data fusion and send the processed data to the sink through other $\mathrm{CHs}$ or sensor nodes. Each sensor node only belongs to one and only one cluster and communicates with its $\mathrm{CH}$. The functionality of a cluster based WSN with single-hop communication inside the clusters. The advantages of a cluster based WSN are as follows. It reduces energy consumption significantly; conserves communication bandwidth and improves the overall scalability of the network.
In this paper, we propose a clustering algorithm for energy efficiency in wireless sensor network. The algorithm is based on game theory. We show that the algorithm outperforms the by simulations. The paper is organized as follows. The related work is presented in Section 2. The proposed algorithm is presented in Section 3. Experimental results are given in Section 4 followed by the conclusion in Section 5.

\section{RELATED WORK}

Clustering algorithms can be classified as Distributed Clustering \& Centralized Clustering. Distributed clustering techniques are further classified into four sub types based on the cluster formation criteria and parameters used for $\mathrm{CH}$ election as Identity based, Neighborhood information based, Probabilistic, and Iterative respectively. Linked Cluster Algorithm (LCA) proposed by [2] belongs to Identity based clustering taking unique node identifiers as key factor to choose cluster heads. Further improvement is provided in terms of LCA2 [2], to eliminate chances of multiple cluster head selection. There are couple of protocols proposed using Neighborhood information based approach. HighestConnectivity Cluster Algorithm (HCCA) [2], is based on choosing a node as cluster head which has highest number of neighbors at 1-hop distance with strict clock synchronization requirements. Max-Min D-Cluster Algorithm [8], selects cluster head in such way that none of its neighbors are d-hop away from it providing better load balancing without clock synchronization requirements. Weighted Clustering Algorithm (WCA) [9], works based on the principle of non-periodic invocation of itself only when topology reconfiguration has become inevitable due to an arbitrary sensor node loosing connectivity with its cluster head while trying to balance combination of several required parameters in the form of common factor called 'combined weight'. Grid-clustering routing Protocol (GROUP) [10], includes multiple sinks with one of them considered as ' primary sink' being responsible for dynamically selecting cluster heads forming grid-like structure.

Probabilistic Approaches for clustering in WSN relies upon prior assigned probability values for sensor nodes. Low-Energy Adaptive Clustering Hierarchy (LEACH) protocol proposed in [1] provides a balancing of energy usage by random rotation of cluster heads meanwhile assuring uniform load balancing in one-hop sensor networks. Two-Level LEACH (TL-LEACH) is discussed in [5], which is an extension to $\mathrm{LEACH}$, proposing primary and secondary level cluster head selection to minimize energy utilization. Energy Efficient Clustering Scheme (EECS) proposes non-iterative, dynamic, and localized competition based process for selection of cluster heads based on residual energy of sensor nodes providing lower message overhead and uniform distribution of cluster heads [6]. Hybrid Energy Efficient Distributed Clustering (HEED) proposes a methodology which takes into account residual energy of sensor 
nodes and intra-cluster communication costs while making selection of cluster heads in multi hop sensor networks [7].

Iterative clustering protocols that need to be mentioned here are: DCA [2], SPAN [4], and ACE [3]. Distributed Clustering Algorithm (DCA) protocol uses delayed willingness announcement technique for any sensor before becoming cluster head thereby giving chance for other higher-weighted (preference conditions) neighbor sensor nodes to have chance of becoming cluster heads. SPAN is a randomized cluster head selection process with localized decision making which is based on number of sensor nodes being benefitted and its own energy levels for a sensor node that is likely to become cluster head. Algorithm for Cluster Establishment is an emergent protocol with two distinct phases of cluster head selection: a randomized new cluster 'spawning phase' and 'migration phase' for existing clusters to achieve highly uniform non-overlapping cluster formation. But, in general, iterative approaches for clustering suffer from their convergence speed dependency upon network diameter.

LEACH-C [17], BCDCP [11], DMSTRP [12], and LEACH-F [13] are protocols of interest in centralized clustering approach. LEACH-C proposes transmission of location awareness and energy levels by each sensor node to base station and sensor nodes with energy level above predetermined threshold are chosen to become cluster heads by base station itself. Base Station Controlled Dynamic Clustering Protocol (BCDCP) also relies on base station for election of cluster heads from a group of sensor nodes by applying residual energy and predetermined energy threshold as a criteria but with a distinction of so elected sensor nodes being capable for operating in 'sensing mode' and 'cluster mode'. Dynamic Minimum Spanning Tree Routing Protocol tries to improve BCDCP behavior by retaining much of its other characteristics. It applies Spanning Tree concept to make optimal decisions about inter-clusters \& intra-clusters. In this regard, DMSTRP turns out to be elegant solution for large networks whereas LEACH-C and BCDCP being better for relatively small networks. LEACH-F is same as LEACH-C as far as cluster head selection is concerned. But, in LEACH-F clusters are fixed once they are formed. Role of cluster head gets rotated for different sensor nodes within each cluster.

\section{THE PROPOSED ALGORITHM BASED ON GAME THEORY}

This section discusses the proposed method, which includes game theory for the energy consumption reduction in Wireless Sensor Networks (WSNs). Each network node has two options i.e. it can or cannot act as cluster head. Since the nodes display a selfish behavior, they do not tend to act as cluster head and assign head clustering to other nodes. In the case that none of nodes acts as cluster head, they cannot send their data to the main station and they cannot gain any utility. The best manner of each node occurs when the node itself is not chosen for cluster heading but at least one of the other residual nodes is introduced as cluster heading. Each node utility gained from the data transmission and cluster heading cost are shown respectively by $\mathrm{V}$ and $\mathrm{C}$. In this case, a two-node network is defined, which shows extent of each node utility per the choice of the node itself and the other node (Table 1). Table 1 can be generalized to multiple-node networks. If we assume that $\mathrm{D}$ and ND stands respectively for the node selecting itself for cluster heading and the node not being regarded the cluster head, then the node is defined on the basis of interaction between the two nodes (table 1).
Table 1: Utility of Interaction between two nodes in a Twonode Network

\begin{tabular}{|c|c|c|}
\hline & ND & D \\
\hline ND & $(0,0)$ & $(\mathrm{v}, \mathrm{v}-\mathrm{c})$ \\
\hline D & $(\mathrm{v}-\mathrm{c}, \mathrm{v})$ & $(\mathrm{v}-\mathrm{c}, \mathrm{v}-\mathrm{c})$ \\
\hline
\end{tabular}

Accordingly, If $S=\{D, N D\}(\mathrm{S}=$ Selection of node i from $\mathrm{S}$ Set), then utility function of each node is defined by Equ. (1):

$$
U_{i}(s)=\left\{\begin{array}{lr}
0 & \text { if } s_{j}=N D \\
v-c & \text { if } s_{i}=D \\
v & \text { if } s_{i}=N D \text { and } s_{j}=D
\end{array}\right.
$$

Therefore, the best manner occurrs when one node is the cluster head and the other nodes are not cluster heads. In this method, each node randomly makes selections in conformity with probability distribution. In the other words, each node has one probability for cluster heading $(\mathrm{P})$ and one probability for noncluster-heading $(q=1-p)$. Utility function value for the latter is estimated on the basis of Equ. (2):

$\mathrm{U}_{\mathrm{ND}}=\mathrm{v} \cdot\left(1-(1-\mathrm{p})^{\mathrm{N}-1}\right)$

The expected utility of non-cluster-heading is regarded equal to cluster heading utility $(\mathrm{v}-\mathrm{c})$ and $\mathrm{P}$ value is measured by Equ. (4):

$\mathrm{v}-\mathrm{c}=\mathrm{v} \cdot\left(1-(1-\mathrm{p})^{\mathrm{N}-1}\right)$

$\mathrm{p}=1-\left(\frac{\mathrm{c}}{\mathrm{v}}\right)^{1 /(\mathrm{N}-1)}$

Then, $\frac{c}{v}$ (a constant number) is regarded equal to W. Equ. (5) is calculated as follows:

$\mathrm{p}=1-\mathrm{w}^{1 /(\mathrm{N}-1)}$

$\mathrm{W}$ is a positive number less than 1 . Accordingly, the $\mathrm{P}$ probability is always between 0 and 1 . By an increase of nodes, $P$ value is reduced. Conversely, by a reduction of nodes, $P$ value is increased. By an increase of nodes, they hardly tend to participate in the network because they show selfish behavior. Therefore, $\mathrm{P}$ probability of each node is calculated by measuting $\mathrm{C}$ and $\mathrm{V}$ and determining the energy modell in ordinary nodes and cluster head nodes. Equa. (6) calculates each node energy consumption in case of data transmission. $\mathrm{K}$ and $\mathrm{d}$ are numbers of the sent bits and the distance between node $\mathrm{i}$ and the cluster head respectively. Hence, each node consumes $E_{t x}(k, d)$ in a distance from d for sending the data bits to the cluster head. Additionally, each node's consumed energy for receiving data is calculated by Equa. (7) (each node consumes Erx (k) for receiving the data bit $\mathrm{K}$ ):

$\mathrm{E}_{\mathrm{tX}}\left(\mathrm{i}, \mathrm{CH}_{\mathrm{i}}\right)=\mathrm{k} \times\left(\mathrm{E}_{\mathrm{elec}}+\varepsilon_{\mathrm{amp}} \times \mathrm{d}_{\mathrm{i}}^{2}\right)$

$\mathrm{E}_{\mathrm{rX}}(\mathrm{k})=\mathrm{E}_{\mathrm{elec}} \times \mathrm{k}$

$E_{\text {elec }}$ and $\varepsilon_{a m p}$ are predefined constants, which are used for clarifying internal circuits of sensors for sending and receiving data as well as other energy consumption operations. When the data are sent from ordinary nodes to cluster heads, the latter aggregate the received data and send them to the main station. If $N_{u}$ is the number of packages which have equal height (k) and are received by the cluster head, then the energy consumed for aggregation of packages is measured by Equa. (8): 
Eaggr $=\mathrm{Nu} \times \mathrm{k} \times$ efuse

$e_{\text {fuse }}$ is a constant used for data aggregation. Moreover, the energy consumed for sending data from cluster heads to the main station is calculated by Equa. (9) $\left(d_{i}\right.$ is the distance between the cluster head $i$ and the main station):

$\mathrm{E}_{\mathrm{tX}}\left(\mathrm{CH}_{\mathrm{i}}, \mathrm{Sink}\right)=\mathrm{k} \times\left(\mathrm{E}_{\mathrm{elec}}+\varepsilon_{\mathrm{amp}} \times \mathrm{d}_{\mathrm{i}}^{4}\right)$

With regard to the above equations, when a node sends its data to the cluster head, it consumes $\mathrm{E}_{\mathrm{tx}}\left(\mathrm{i}, \mathrm{CH}_{\mathrm{i}}\right)$. The cluster headreceives the sent data from the member nodes and sends them to the main station after their aggregation. Hence, the cluster head node cost (c) is estimated by Equa.(10):

$$
\begin{array}{r}
\mathrm{E}_{\mathrm{tX}}\left(\mathrm{CH}_{\mathrm{i}}, \text { Sink }\right) \times \mathrm{c}=\mathrm{N}_{\mathrm{u}} \times \mathrm{E}_{\mathrm{rx}} \times \mathrm{E}_{\mathrm{aggr}}(10) \\
\mathrm{c}>\mathrm{E}_{\mathrm{tX}}\left(\mathrm{i}, \mathrm{CH}_{\mathrm{i}}\right)=\mathrm{t}
\end{array}
$$

If $\alpha$ and $\beta$ are data transmission rate and data receipt rate respectively, then the exchanged energy for each node $\left(E_{D}\right)$ is calculated by Equa. (11). The resulted utility (v) is measured by Equa. (12):

$\mathrm{E}_{\mathrm{D}}=\alpha \cdot \mathrm{E}_{\mathrm{tx}}\left(\mathrm{i}, \mathrm{CH}_{\mathrm{i}}\right)+\beta \cdot \mathrm{E}_{\mathrm{rX}}$

$\mathrm{v}=(\alpha+\beta) \times \mathrm{E}_{\mathrm{tx}}-\mathrm{ED}$

Accordingly, the probability of node cluster heading (p) is measured as follows:

$\mathrm{p}=1-\left(\frac{\mathrm{c}-\mathrm{t}}{\mathrm{v}-\mathrm{t}}\right)^{1 /(1-\mathrm{N})}$

The members of each cluster (cluster heads) are selected based on their distance to the nearest cluster or clustre head.

\section{EXPERIMENTAL RESULTS}

In this section, we evaluate the performance of our algorithm via simulations. We assume that the base station is fixed and

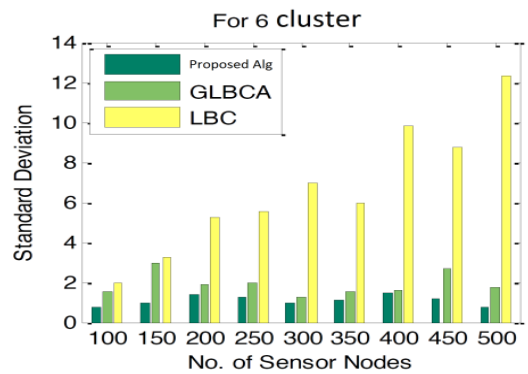

(a)

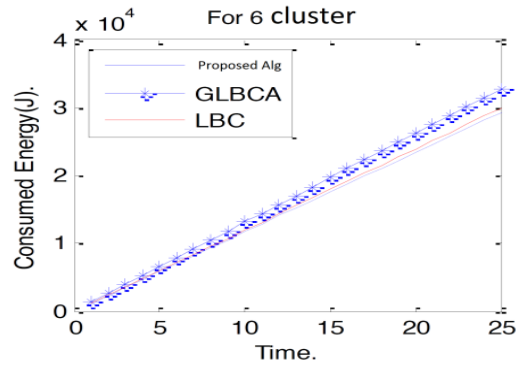

(c) located far away from the network. The experiments were performed using MATLAB on an Intel Core 2 Duo processor with T9400 chipset, $2.53 \mathrm{GHz}$ CPU and $2 \mathrm{~GB}$ RAM running on the platform Microsoft Windows 7. The experiments are performed with diverse number of nodes placed in a $1000 \mathrm{X}$ 1000 square meter area by varying the number of sensor nodes from 100 to 500 and the number of $\mathrm{CHs}$ from 4 to 10 . Each sensor node is assumed to have an initial energy of 2 joules. A node is considered dead if its energy level reaches to 0 joules.

For the sake of comparison, we also ran the Chor Ping Low's approximation algorithm GLBCA (Chor Ping Low et al., 2008) and Gaurav Gupta's algorithm LBC (Gaurav Gupta et al., 2012). In order to judge the quality of the load balancing, we measure the standard deviation of the loads of the $\mathrm{CHs}$ and plot against the number of sensor nodes. It can be observed that our proposed algorithm is better than GLBCA and far better than LBC as shown in Figure 1(a). We also obtain the execution time for run of the same experiments. As shown in Figure 1(b) that the proposed algorithm is better than LBC and far better than GLBCA in terms of execution time. In Figure 1(c) and $2(d)$, we show energy $(\mathrm{J})$ consumption and number of dead sensor node against per round respectively. It is observed that our proposed algorithm outperforms the GLBCA and LBC in terms of energy consumption and number of sensor nodes dies too.

\section{CONCLUSION}

One of WSN problems is energy resource limitations. This study introduces a new method on the basis of game theory. The simulation findings reveal that the proposed algorithm can put in a more proper performance and prolong the network life as compared with similar algorithms. In the proposed method, the basic station is static. Therefore, future studies can concentrate on dynamicity of basic station. Moreover, the proposed method is a centralized protocol in which clusters are formed by the basic station. Future studies can design distributed-environment-related algorithms.

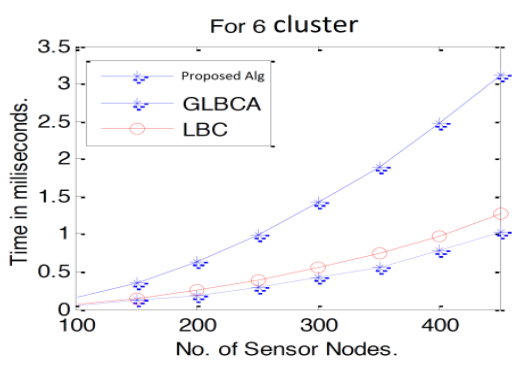

(b)

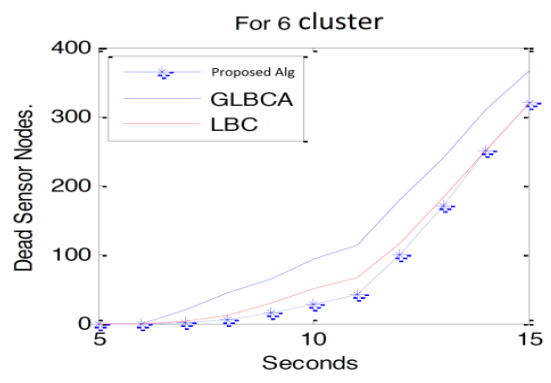

(d)

Figure 1. Comparison between GLBCA, LBC and our proposed algorithm in terms of (a) Load balancing, (b) Execution time, (c) Consumed Energy (J) and (d) Number of Sensor nodes dies 


\section{REFERENCES}

[1] Wendi Rabiner, Heinzelman, Anantha Chandrakasan, HariBalakrishnan. Energy- Efficient Communication Protocol for Wireless Microsensor Networks. Proceedings of IEEE 2000.

[2] P. Kumarawadu, D. J. Dechene, M. Luccini, A. Sauer Algorithms for Node Clustering in Wireless Sensor Networks: A Survey. Proceedings of IEEE 2008.

[3] Haowen Chan, Adrian Perrig. ACE: An Emergent Algorithm for Highly Uniform Cluster Formation. Proceedings of the First European Workshop on Sensor Networks (EWSN), Vol. 2920 Springer (2004) , p.154171

[4] Benjie Chen, Kyle Jamieson, HariBalakrishnan, Robert Morris. Span: An Energy Efficient Coordination Algorithm for Topology Maintenance in Ad Hoc Wireless Networks. Wireless Networks 8, 481-494,2002, Kluwer Academic Publishers.

[5] V. Loscrì, G. Morabito, S. Marano.: A Two-Levels Hierarchy for Low-Energy Adaptive Clustering Hierarchy (TL-LEACH). Proceedings of IEEE 2005, 0-7803-9152$7 / 05$.

[6] Mao Ye1, Chengfa Li, Guihai Chen1, Jie Wu. EECS: An Energy Efficient Clustering Scheme in Wireless SensorNetworks. 24th IEEE International Performance, Computing, and Communications Conference, 2005. IPCCC 2005.

[7] OssamaYounis and Sonia Fahmy. HEED: A Hybrid, Energy-Efficient, Distributed Clustering Approach for Adhoc Sensor Networks. IEEE transactions on Mobile computing, Vol 3, No 4, Oct-Dec 2004.

[8] Alan D. Amis, Ravi Prakash, Thai H.P., Vuong Dung, T. Huynh. Max-Min D-Cluster Formation in Wireless AdHoc Networks. Proceedings of IEEE conference INFOCOM 2000 .

[9] Maniakchatterjee, Sajal. K.das, DamlaTurgut. WCA: A Weighted Clustering Algorithm for wireless adhoc networks. Journal of cluster computing (Special issue on Mobile AdHoc Networks) 2002.

[10] Liyang Yu, Neng Wang, Wei Zhang, Chunlei Zheng. GROUP: a Grid-clustering Routing Protocol for Wireless Sensor Networks. Proceedings of IEEE conference on Wireless communications, Networking and Mobile Computing (WiCOM), 2006.

[11] Manjeshwar A., Agrawal D. P. 2002. APTEEN: a hybrid protocol for efficient routing and comprehensive information retrieval in wireless sensor networks. in: Proc. of International Parallel and Distributed Processing Symposium (IPD , pp.195-202, Fort

[12] Lauderdale, Florida (April 2002) Amir Sepasi Zahmati et al., 2007. Energy-Efficient Protocol with Static Clustering for Wireless Sensor Networks, World Academy of Science, Engineering and Technology, Vol. 28, pp.69-72.

[13] Bencan Gong et al., 2008. Multihop Routing Protocol with unequal Clustering for Wireless Sensor Networks, International Colloquium on Computing, Communication, Control, and Management, (ISECS2008), Vol. 2, pp. 552556

[14] Pratyay K., Prasanta K. J., 2012. Improved Load Balanced Clustering Algorithm for Wireless Sensor Networks, ADCONS 2011, LNCS, Vol. 7135, pp. 399-404.

[15] Zhixin Liu et al. 2011. A distributed energy-efficient clustering algorithm with improved coverage in wireless sensor networks, Future Generation Computer Systems, pp. 1-11.

[16] Xiang Min et al., 2010. Energy efficient clustering algorithm for maximizing lifetime of wireless sensor networks, Int. J. Electronics Communication. (AEÜ), Vol. 64, pp. 289-298.

[17] Wei Li, 2009. Energy Efficient Clustering Algorithm in Wireless Sensor Networks based on Geometric Programming. 2nd International Symposium on Electronic Commerce and Security, IEEE, Vol. 2, pp. 525-529. 